\title{
Aspectos sensório-orais e suas interferências no comportamento alimentar da criança
}

\author{
Patrícia Junqueira ${ }^{1}$
}

\begin{abstract}
Resumo
Dificuldades alimentares na infância são extremamente comuns. Estudos realizados em diferentes regiões do mundo mostram que a queixa de não comer está entre as principais situações, não apenas nos consultórios de pediatras como também na clínica de especialistas, como gastroenterologistas e fonoaudiólogos. Por ser uma alteração complexa e com etiologias diversas, as dificuldades alimentares necessitam de uma abordagem multiprofissional, que seja capaz de identificar e tratar todos os aspectos envolvidos na refeição da criança. Aspectos sensório-motores orais alteram o comportamento alimentar da criança. O papel do fonoaudiólogo é identificar se há e quais dessas interferências estão impactando nesse processo. Cabe ainda prever uma abordagem holística que não enfoque apenas a cavidade oral, mas todos os aspectos que compõem e se inter- relacionam no momento da refeição. Desse modo estará colaborando para o desenvolvimento de uma alimentação saudável e prazeirosa para todos: a criança e sua família.
\end{abstract}

Palavras chave: Terapia Alimentar1; Comportamento Alimentar2; Defesa Sensorial3

Junqueira, Patrícia. "Aspectos sensório-orais e suas interferências no comportamento alimentar da criança", in Anais do 2‥ Congresso Internacional Sabará de Especialidades Pediátricas

\footnotetext{
${ }^{1}$ Fonoaudióloga. Doutora em Distúrbios da Comunicação Humana- UNIFESP

Responsável pelo Serviço de Fonoaudiologia do Centro de Dificuldades Alimentares Infantis do Instituto de Pesquisa e Ensino em Saúde Infantil - PENSI- Hospital Infantil Sabará, São Paulo, SP, Brasil. patriciajunqueiram@uol.com.br
} 
Dificuldades alimentares na infância são extremamente comuns. Estudos realizados em diferentes regiões do mundo mostram que a queixa de não comer está entre as principais situações, não apenas nos consultórios de pediatras como também na clínica de especialistas, como gastroenterologistas e fonoaudiólogos.Diferentes levantamentos científicos mostram que o problema acomete de $8 \%$ a $50 \%$ das crianças, dependendo dos critérios diagnósticos utilizados, independentemente de idade, sexo, etnia e condição econômica Para a família, as dificuldades alimentares representam uma das preocupações mais importantes enfrentadas na infância, sendo causa frequente de conflitos nas relações entre pai, mãe e filhos.

Na tentativa de auxiliar o entendimento dos diferentes aspectos do problema, Kerzner sugere a expressão "dificuldade alimentar" como nomenclatura geral e propõe uma classificação baseada em características de apresentação do quadro, organizada em sete perfis: interpretação equivocada dos pais, ingestão altamente seletiva, criança agitada com baixo apetite, fobia alimentar, presença de doença orgânica, criança com distúrbio psicológico ou negligenciada e choro que interfere na alimentação. As dificuldades alimentares apresentam, ainda, a característica de serem distúrbio duradouro, que se apresenta nos primeiros meses de vida e persiste durante muitos anos, conforme demonstrado por Ostberg\&Hagelin, que acompanharam 230 famílias suecas e verificaram que as crianças com essa condição nos primeiros anos de vida persistiam com problemas nessa área após seis anos de seguimento.

Por ser uma alteração complexa e com etiologias diversas, as dificuldades alimentares necessitam de uma abordagem multiprofissional, com participação do pediatra, nutricionista, fonoaudiólogo, psicólogo, terapeuta ocupacional e gastropediatra, entre outros. Os fonoaudiólogos há muito tempo atuam com bebês e crianças com dificuldades alimentares, com o objetivo de "fazer a criança comer" uma quantidade específica e/ou em qualidade mais adequada em termos de textura e consistência do alimento. Para alcançar esses objetivos,foram desenvolvidos programas de tratamento da motricidade orofacial que enfatizavam a estimulação e os movimentos da mandíbula, língua e lábios, com o objetivo de adequar essas estruturas para que a criança assim pudesse se alimentar. Esse modo de atuação tem um conceito restrito subjacente que permeia esse tipo de terapia, no qual o grande objetivo é melhorar as habilidades motoras orais da criança para que ela possa comer. Nessa visão a lente do fonoaudiólogo se fecha num enorme zoom para a criança caracterizando o modo de olhar e tratar as dificuldades alimentares infantis.

De acordo com Morris para melhor compreensão das dificuldades alimentares infantis é necessário a análise da problemática sob novo ângulo, porque quando se abre o foco, ampliando o campo de visão, outros aspectos são observados e o fonoaudiólogo tem uma compreensão mais ampla do momento da refeição e não apenas da dificuldade da criança.

Passa-se a apreciar e compreender os relacionamentos que se estabelecem durante a refeição e como os mesmos são expressos e influenciados pela comunicação, aprendizagem, aspectos físicos e sensoriais que compõem e fazem parte desse momento.

Esses aspectos compõem o Círculo da Refeição descritos por Morris. FIG 1. 


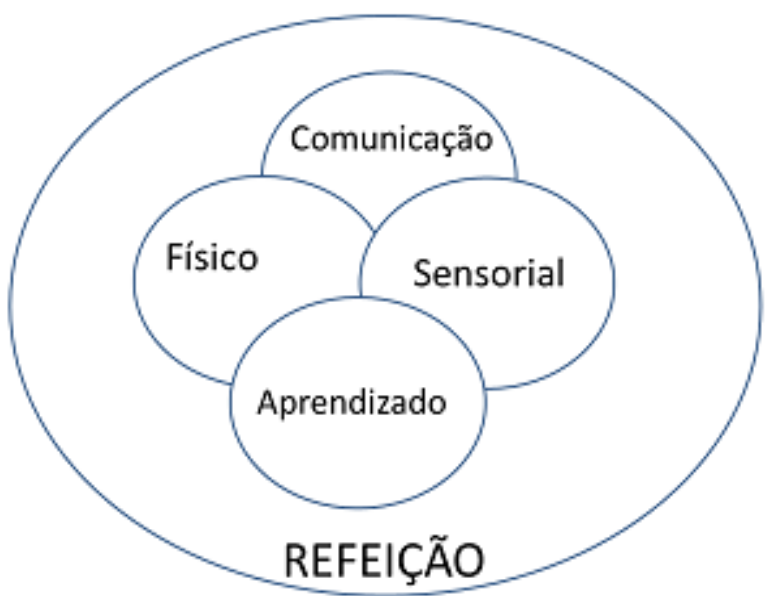

Fig 1- Círculo da Refeição

Pre-feeding skills:acomprehensive resource for mealtime development. Morris SE, Klein MD. 2nd ed. Pro-ed 2000.

Esse círculo representa a total interação e influência sob a refeição da criança. Ele inclui todos os aspectos dos quatro ciclos fechados que estão interconectados. Em adição ele incorpora o amplo aspecto do relacionamento da criança com a sua família na refeição.

Algumas crianças vivenciam uma quebra nesse ciclo normal de desenvolvimento devido as dificuldades físicas (motoras orais) e sensoriais.

Normalmente essas dificuldades surgem nos momentos de transição alimentar. Ao iniciar a introdução da mamadeira, alguns bebês podem apresentar dificuldades em aceitar o bico. Outros momentos importantes são o início da primeira papa, a introdução de grãos, e a passagem para a alimentação usual da família.

As famílias usualmente respondem a essas dificuldades da criança com alimentação ou recusa alimentar com pressão na tentativa de controlar o tipo e a quantidade de alimento que a criança come.

Muitas vezes sem compreender os motivos pelos quais a criança não aceita determinado alimento, ou ainda sem uma real compreensão da dificuldade apresentada, começam a travar verdadeiras "batalhas" para que a criança coma. Esse comportamento dos pais contribui para uma relação desfavorável entre todos os envolvidos no momento da refeição, desgastando as relações estabelecidas nesse momento, além de criar uma negativa associação entre alimento e desejo de comer.

Desse modo, identificar os bloqueios que limitam o desenvolvimento das habilidades para comer com conforto, é essencial para ajudar as crianças a retornarem seu desejo natural de participar do momento da refeição

Os aspectos físicos e motores- orais envolvidos no ato de comer e beber quando alterados aumentam a tensão e limitam os padrões de movimentos da boca, podendo facilitar padrões de movimentos automáticos ou reflexos orais. Podem também reduzir o controle da cabeça e tronco que proporciona a estabilidade postural e suporte dos movimentos orais.

Os aspectos sensoriais exercem grande papel em determinar o tipo de movimento que será utilizado ou se a criança vai explorar a experiência de aprendizagem de novos padrões de movimento para a alimentação.

Compreendendo as habilidades sensoriais normais como:

1-Capacidade dos órgãos sensoriais receberem a informação sensorial ; 2-Habilidade do SNC interpretar e ou perceber a mensagem sensorial; e 3- Controle da quantidade total de 
informação que está sendo processada, qualquer desvio em uma dessas habilidades compromete o desenvolvimento do processo da alimentação.

Isso acontece porque uma disfunção sensorial pode aumentar ou diminuir o nível de intensidade de sensações ao comer promovendo desconforto; causar sensações associadas ao alimento que são percebidas como extremamente desagradáveis, perigosas e ameaçadoras, limitando o número de alimentos que a criança come, contribuindo para a recusa alimentar.

Os reflexos dessa disfunção na integridade sensorial acarretam mudanças no comportamento da criança que nem sempre são compreendidos pelos pais. Muitas crianças não toleram sentar a mesa, outras fogem ao ver determinados alimentos, outras desenvolvem fobia extrema a determinados tipos de preparação.

O papel do fonoaudiólogo é identificar se há, e quais dessas possíveis interferências estão impactando no desenvolvimento alimentar da criança. Prever uma abordagem holística que não foque apenas a cavidade oral, mas todos os aspectos que compõem e se inter- relacionam no momento da refeição.

Desse modo estará colaborando para o desenvolvimento de uma alimentação saudável e prazeirosa para todos: a criança e sua família.

Oral motor sensory issues and their interference in the child's feeding behavior

Abstract:. Feeding difficulties in infancy are extremely common. Studies conducted in different regions of the world show that the complaint of not eating is among the leading situations, not just in pediatricians in the clinic as well as specialists such as gastroenterologists and speech language pathologists. To be a complex and diverse etiologies change, food difficulties require a multidisciplinary approach, which is able to identify and treat all aspects involved in the child's meal. Aspects of oral sensorimotor alter the feeding behavior of the child. The role of the speech therapist is to identify if there is interference and which of these are impacting this process. It should also provide for a holistic approach that not only the oral cavity, but all aspects that comprise and interrelate at meal time. Thus will be collaborating for the development of a healthy and enjoyable food for all: the child and his family.

Key words:1- Feeding therapy, Feeding behavior, Oral motor disorders

\section{Referencias}

Almeida CN, Mello ED, Maranhão HS, Vieira MC, Barros R, Barreto JR et al. Dificuldades alimentares na infância: revisão da literatura com foco nas repercussões à saúde. PediatriaModerna. 2012;48(9):24-6. 
Brown AM, Matheny AP, Jr. Feeding problems and preschool intelligence scores: a study using the co-twin method. Am J ClinNutr. 1971;24(10):1207-9.

Carruth BR, Ziegler PJ, Gordon A, Barr SI. Prevalence of picky eaters among infants and toddlers and their caregivers, and decisions about offering a new food. J Am Diet Assoc. 2004;104:57-64

Chatoor I, Surles J, Ganiban J, Beker L, Paez LM, Kerzner B. Failure to thrive and cognitive development in toddlers with infantile anorexia. Pediatrics. [Research Support, U.S. Gov't, P.H.S.]. 2004;113(5):e440-7.

Jacobi C, Agras WS, Bryson S, Hammer LD. Behavioral validation, precursors, and concomitants of picky eating in childhood. J Am Acad Child Adolesc Psychiatry. [Research Support, Non-U.S. Gov’t Research Support, U.S. Gov’t, P.H.S.]. 2003;42(1):76-84.

Kerzner B. Clinical investigation of feeding difficulties in young children: a practical approach. ClinPediatr (Phila). [Review]. 2009;48(9):960-5.

Marchi M, Cohen P. Early childhood eating behaviors and adolescent eating disorders. J Am Acad Child Adolesc Psychiatry. [Research Support, Non-U.S. Gov't Research Support, U.S. Gov't, P.H.S.]. 1990;29(1):112-6.

Mascola AJ, Bryson SW, Agras WS. Picky eating during childhood: a longitudinal study to age 11 years. Eat Behav. [Research Support, N.I.H., Extramural]. 2010; 11(4):253-7.

Morris SE, Klein MD Pre-feeding skills:acomprehensive resource for mealtime development.. 2nd ed. Pro-ed 2000.

Östberg M, Hagelin E. Feeding and sleeping problems in infancy: a follow-up at early school age. Child: Care, Health and Develop. 2011;37(1):11-25.

Overland L .A sensory-motor approach to feeding.._SIG 13 Perspectives on swallowing and swallowing disorders (dysphagia).2011;(20):60-4. doi:10.1044/sasd20.3.60

Rommel N, Meyer A M, Feenstra L, Veereman- Wauters,G. The complexity of feeding problems in 700 infants and young children presenting to a Tertiary Care Institution. JPediatGastroentNutrit. 2003; (37):75-84.

Satter E. How to get your kid to eat, But not too much. 1987.Bull Publishing Company, 1987.

Sheppard JJ. Using motor learning approaches for treating swallowing and feeding disorders: a review. Language, speech, and hearing services in schools.2008;(39): 227-36. doi:10.1044/01611461(2008/022)

William J. Roche; Peggy $\quad$ S. Eicher; Pamela Martorana; Merrill Berkowitz; JoAnn Petronchak; Jessica Dzioba; Louise VitelloAn Oral, Motor, Medical, and Behavioral Approach to Pediatric Feeding and Swallowing Disorders: An Interdisciplinary Model. Perspectives on Swallowing and Swallowing Disorders (Dysphagia) October 2011, Vol.20, 65-74. doi:10.1044/sasd20.3 